\title{
End-grafted sugar chains as aqueous lubricant additives Synthesis and macrotribological tests of poly(l-lysine)- graft-dextran (PLL-g-dex) copolymers
}

\author{
Journal Article \\ Author(s): \\ Perrino, Chiara; Lee, Seunghwan; Spencer, Nicholas D. \\ Publication date: \\ 2009 \\ Permanent link: \\ https://doi.org/10.3929/ethz-b-000015401 \\ Rights / license: \\ In Copyright - Non-Commercial Use Permitted \\ Originally published in: \\ Tribology Letters 33(2), https://doi.org/10.1007/s11249-008-9402-6
}




\title{
End-grafted Sugar Chains as Aqueous Lubricant Additives: Synthesis and Macrotribological Tests of Poly(L-lysine)-graft- Dextran (PLL-g-dex) Copolymers
}

\author{
Chiara Perrino $\cdot$ Seunghwan Lee $\cdot$ Nicholas D. Spencer
}

Received: 19 September 2008/ Accepted: 8 December 2008/Published online: 30 December 2008

(C) Springer Science+Business Media, LLC 2008

\begin{abstract}
Comb-like graft copolymers with carbohydrate side chains have been developed as aqueous lubricant additives for oxide-based tribosystems, in an attempt to mimic biological lubrication systems, whose surfaces are known to be covered with sugar-rich layers. As adopted in the previous studies of the graft copolymer poly(L-lysine)graft-poly(ethylene glycol) (PLL-g-PEG), which showed both excellent lubricating and antifouling properties, a similar approach was chosen to graft dextran chains onto the same backbone, thus generating PLL- $g$-dex. PLL- $g$-dex copolymers readily adsorb from aqueous solution onto negatively charged oxide surfaces. Tribological characterization at the macroscopic scale, either under pure sliding conditions or a mixed sliding/rolling contact regime, shows that PLL- $g$-dex is very effective for the lubrication of oxide-based tribosystems. The relative lubricating capabilities of PLL-g-dex copolymers compared with PLL- $g$-PEG copolymers were observed to be highly dependent on the molecular structure of the copolymers (in particular, sidechain density along the backbone) and the measurement conditions (in particular, time between tribocontacts); the PLL-g-dex copolymers with a low degree of grafted side chains $(\leq 20 \%$ grafting of available protonated primary amine groups along the backbone) showed better lubricating performance than their PLL- $g$-PEG counterparts at high tribocontact frequency ( $\geq$ ca. $0.32 \mathrm{~Hz}$ ).
\end{abstract}

Keywords Aqueous lubrication - Boundary lubrication . Biomimetic lubricant additives · Poly(L-lysine)-g-dextran

C. Perrino $\cdot$ S. Lee $\cdot$ N. D. Spencer $(\varangle)$

ETH Zurich, Wolfgang-Pauli-Strasse 10,

CH 8093 Zurich, Switzerland

e-mail: nicholas.spencer@mat.ethz.ch

\section{Introduction}

Water-based lubricants possess distinctive advantages over oil-based lubricants (such as environmental compatibility, biocompatibility, availability, cost effectiveness), and could represent a viable alternative in certain applications. Their environmental compatibility makes them suitable for a number of industrial applications, such as food processing or textile and pharmaceutical manufacturing, where the use of oil-based lubricants can be problematic due to contamination issues $[1,2]$. In the biomedical field, aqueous lubrication is particularly important, since water is virtually the only acceptable base lubricant. Any attempts to improve lubrication properties in biomedical applications (such as lubricious coatings for catheters) should therefore take both water compatibility and biocompatibility into account [3].

Poly(L-lysine)-graft-poly(ethylene glycol) (PLL-g-PEG), a graft copolymer consisting of a polycationic PLL backbone and PEG side chains, has proven to be highly effective on oxide surfaces with regard to both preventing non-specific adsorption of proteins and lubricating in an aqueous environment [4-11]. PLL- $g$-PEG has been shown to spontaneously adsorb from aqueous solutions onto negatively charged surfaces via electrostatic interactions; the positive charges present on the protonated primary amine groups of the PLL backbone in a neutral aqueous environment lead to its rapid immobilization onto negatively charged surfaces. PEG side chains, which are radially distributed along the backbone in bulk solution in order to minimize steric repulsion, stretch out into the solution just after the copolymers have adsorbed on the surface, forming a polymer brush, providing the inter-PEG spacing is sufficiently close. PEG, however, has some disadvantages, such as lower solubility with increasing 
temperature and oxidation to peroxides in air [12, 13], which could limit the usage of PLL- $g$-PEG in certain applications. An alternative approach is to mimic biological membrane surfaces, which are typically covered with carbohydrate-rich layers. In vivo cellular interactions can be strongly influenced by pendant oligosaccharides chains, the principal components of the highly hydrated glycocalyx, which surrounds certain kinds of cell, and is known to prevent undesirable, non-specific adsorption of proteins [14-18]. Carbohydrates are also known to play an important role in natural lubrication mechanisms, which often involve a brush-like structure of sugar chains, anchored to a protein backbone. Proteoglycans, for instance, have been suggested to play a role in natural joint lubrication $[19,20]$, and mucins, large glycoproteins that make up the mucosal hydrogel coating covering epithelial cell surfaces, act as both protective and lubricious layers [21-24].

Based on this natural model, sugar-based copolymers have been developed for use as aqueous lubricant additives. In this study, encouraged by the successful lubricating performance of PLL- $g$-PEG in previous studies [6-8], we have chosen to maintain the comb-like graft polymer structure, selecting the same PLL backbone, and replacing the PEG side chains with dextran chains (Fig. 1). Dextran is a natural and neutral polysaccharide consisting of an $\alpha(1 \rightarrow 6)$-linked glucan with side chains attached to the 3 -positions of the backbone glucose units. Our choice of dextran for the carbohydrate chains was motivated by several factors. First, there are already reports on the synthesis and applications for drug/gene delivery of poly (L-lysine)$g$-dextran (PLL-g-dex) in the literature [25-31]. Second, since the dextran is neutral, it does not present problems due to electrostatic interactions when grafted onto a polycationic backbone (PLL). Finally, previous studies have shown the effectiveness of dextran coatings at preventing non-

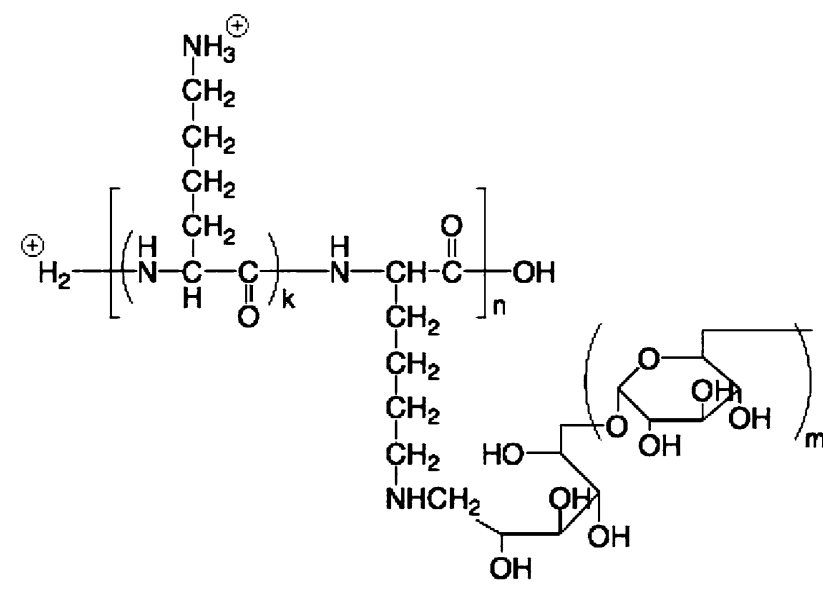

Fig. 1 A schematic illustration of the chemical structure of PLL- $g$-dextran. $\mathrm{k}$ should be taken as an average value. $\mathrm{k}+1$ represents the grafting ratio of the polymer specific protein adsorption [14-18, 32-40], this being a property that often seems to be closely associated with good aqueous lubricating properties [5, 6, 41, 42].

We have focussed on the characterization of adsorption and aqueous lubricating properties of PLL-g-dex copolymers on oxide surfaces under standard, macroscopic tribological conditions at ambient temperatures, including sliding and mixed sliding/rolling contacts. In particular, we have synthesized and employed various PLL-g-dex copolymers with varying architectural features, including the molecular weight and the grafting ratio of the dextran side chains along the backbone, to characterize the relative lubricating capabilities compared with counterpart PLL- $g$-PEG copolymers.

\section{Experimental}

\subsection{Poly(L-lysine)-graft-dextran (PLL-g-dex)}

Poly(L-lysine)-graft-dextran (PLL-g-dex) copolymers were synthesized by a reductive amination reaction of PLL $\mathrm{HBr}$ (20 kDa, polydispersity 1.1 , Sigma-Aldrich, Switzerland) with dextran (dextran T5, 5.2 and T10, $10 \mathrm{kDa}$, polydispersity 1.8, Pharmacosmos A/S, Denmark). Borate buffer (50 mM, pH 8.5) was used as solvent for the reaction. The reaction proceeded in two steps: first, the formation of a Schiff base between the terminal dextran aldehyde group and primary amine groups of PLL, and second, the reduction of the unstable Schiff base to secondary amines. PLL was dissolved in borate buffer solution at $40{ }^{\circ} \mathrm{C}$, and dextran was subsequently added to the solution, and the mixture was incubated with stirring overnight for the formation of the Schiff base. Sodium cyanoborohydride $\left(\mathrm{NaBH}_{3} \mathrm{CN}\right.$, Fluka Chemika, Switzerland) was then added to the mixture $(10 \times$ molar excess to dextran was used $)$ and it was kept under stirring for two more days. The resulting copolymers were isolated by ultracentrifugation (Vivaspin 15R centrifugation tubes, 30000 MWCO, Sartorius AG, Switzerland) to remove the unreacted starting materials. Varying the ratio of Lys/dex allowed us to control the degree of grafting of dextran chains onto the PLL backbone (see below for the definition of the grafting ratio, $g$ ). A 2-3 $\times$ molar excess of dextran was required for the synthesis to obtain a given grafting degree. PLL- $g$-dex molecules were characterized by ${ }^{1} \mathrm{H}-\mathrm{NMR}$ spectroscopy and elemental analysis (EA). ${ }^{1} \mathrm{H}-\mathrm{NMR}$ spectra of the copolymers in $\mathrm{D}_{2} \mathrm{O}$ were recorded on a Bruker spectrometer $(300 \mathrm{MHz})$ and both NMR spectra and elemental analysis data were used to determine the grafting ratio.

The notation PLL $(x)-g[y]-\operatorname{dex}(z)$ for the copolymers was used to represent the molar mass of PLL in $\mathrm{kDa}(x)$ (including the counterions, $\mathrm{Br}-$, as precursor), the molar 
mass of dextran in $\mathrm{kDa}(z)$, and the grafting ratio $g[y]$ (defined as the number of lysine monomers/dextran side chain). Although it is possible, in principle, to control all three structural parameters, we have varied only the grafting ratio, $y$, and the molar mass of dextran, $z$, whereas the molecular weight of PLL was kept constant at $20 \mathrm{kDa}$, in this study. For comparison purposes, PLL(20)-g-PEG(5) copolymers provided by SuSoS AG (Dübendorf, Switzerland) have also been investigated, with PEG(5) side chains (molecular weight, $5 \mathrm{kDa}$ ) on a PLL(20) backbone and covering a similar range of grafting ratios to that of the PLL-g-dex copolymers employed [5].

\subsection{Optical Waveguide Lightmode Spectroscopy (OWLS)}

Optical waveguide lightmode spectroscopy (OWLS) was used to characterize the adsorption properties of the polymers. Experiments were performed using an OWLS 110 instrument (Microvacuum, Budapest, Hungary).

OWLS is an optical biosensing technique for the in situ label-free analysis of adsorption processes [43]. In brief, linearly polarized light (He-Ne laser) is coupled with a diffraction grating into a planar waveguide, provided that the in-coupling condition is fulfilled. The light is guided by total internal reflection to the ends of the waveguide layer, where it is detected by a photodiode detector. The adsorbed mass is calculated from the change in the refractive index in the vicinity of the waveguide surface upon adsorption of molecules [43-45]. The refractive index increment $(\mathrm{dn} / \mathrm{dc})$ of dextran was measured by means of a refractometer and a value of 0.131 was used for all measurements to calculate the mass of polymer adsorbed. For the measurement of the mass of adsorbed PLL- $g$-PEG copolymer, a value of 0.139 was always used.

Prior to the experiments, optical waveguides chips (standard: $\mathrm{Si}_{0.75} \mathrm{Ti}_{0.25} \mathrm{O}_{2}$ on glass, $1.2 \times 0.8 \mathrm{~cm}^{2}$, Microvacuum, Budapest, Hungary) were ultrasonicated in $0.1 \mathrm{M}$ $\mathrm{HCl}$ for $10 \mathrm{~min}$, rinsed with Millipore water, ultrasonicated in 2-propanol for $10 \mathrm{~min}$, rinsed again with Millipore water, and then dried under a dry nitrogen stream. The substrates were subsequently cleaned by UV/ozone cleaner (Boeckel industries Inc., model 135500) for $30 \mathrm{~min}$.

The cleaned waveguides were assembled into the OWLS flow cell and equilibrated by exposing to HEPES buffer solution (10 mM 4-(2-hydroxyethyl)piperazine-1ethanesulfonic acid (Sigma, St. Louis, MO, USA), adjusted to $\mathrm{pH} 7.4$ with $6.0 \mathrm{M} \mathrm{NaOH}$ solution) overnight in order to obtain a stable baseline. The waveguides were then exposed to a polymer solution $\left(0.25 \mathrm{mg} \mathrm{mL}^{-1}\right.$ in HEPES buffer) for at least $30 \mathrm{~min}$, resulting in the formation of a polymer adlayer, and rinsed three times by soaking them in buffer solution for another $30 \mathrm{~min}$.

\subsection{Pin-on-disk Tribometry}

A pin-on-disk tribometer (CSM Instruments, Switzerland) was employed to investigate the lubricating properties of the polymer solutions under pure sliding conditions.

As a tribopair, we chose steel balls $(6 \mathrm{~mm}$ in diameter, DIN 5401-20 G20, Hydrel AG, Romanshorn, Switzerland) against flat glass squares $\left(2.5 \times 2.5 \mathrm{~cm}^{2}, 1-\mathrm{mm}\right.$ thick $)$, cut from microscope slides (Medite Medizintechnik AG, Switzerland, approximate chemical composition according to the manufacturer: $72.2 \% \mathrm{SiO}_{2}, 14.3 \% \mathrm{Na}_{2} \mathrm{O}$, traces of $\mathrm{K}_{2} \mathrm{O}, \mathrm{CaO}, \mathrm{MgO}, \mathrm{Al}_{2} \mathrm{O}_{3}, \mathrm{Fe}_{2} \mathrm{O}_{3}, \mathrm{SO}_{3}$ ). The lubricating properties of the polymers were evaluated by acquiring the coefficient of friction, $\mu$ (= friction force/load), as a function of speed under a fixed load $(2 \mathrm{~N}$, dead weights) at room temperature. The maximum Hertzian contact pressure in this configuration is estimated to be $0.34 \mathrm{GPa}$. The sliding speed was controlled to vary from 1 to $19 \mathrm{~mm} \mathrm{~s}^{-1}$ using the instrument's software (InstrumX version 2.5A, CSM Instruments, Switzerland). The weight concentration of the polymer solutions was chosen in order to attain similar molar concentrations for all copolymers $\left(0.25 \mathrm{mg} \mathrm{mL}^{-1}\right.$ for PLL-g-dex(5.2) and PLL- $g$-peg(5) copolymers and $0.5 \mathrm{mg} \mathrm{mL}^{-1}$ for PLL-g-dex(10)) with a similar range of grafting ratios as used in the OWLS experiments. The number of rotations was fixed at 200 . For each measurement with a given copolymer, the same pair of pin and disk was used. However, the position of the ball and the radius of the track on the disk were changed for each speed, to provide fresh tribocontact points. Balls and disks were cleaned immediately before each measurement as follows: they were ultrasonicated in ethanol for $10 \mathrm{~min}$, dried under a nitrogen stream and then oxygen-plasma cleaned for $2 \mathrm{~min}$. All instrumental parts expected to be in contact with the polymer solution were cleaned by rinsing with ethanol and water.

\subsection{Mini-traction Machine}

A mini-traction machine (MTM, PCS Instruments, London, UK) was employed to characterize the lubricating behavior of the copolymer solutions in a mixed sliding/rolling contact regime over a speed range of $0-2,500 \mathrm{~mm} \mathrm{~s}^{-1}$. AISI 52100 steel balls $(9.5 \mathrm{~mm}$ in radius, PCS instruments, London, UK) and flat silicate glass disks $(46 \mathrm{~mm}$ in diameter, PCS Instruments, London, UK) were used as tribopairs. The ball is loaded against the disk and ball and disk driven independently to create a mixed sliding/rolling contact. The slide/roll ratio (SRR) is defined as the percentage ratio of the difference to the mean of the ball speed $\left(\mathrm{u}_{\text {ball }}\right)$ and disk speed $\left(\mathrm{u}_{\mathrm{disk}}\right)$; i.e., $\mathrm{SRR}=\left[\left|\mathrm{u}_{\text {ball }}-\mathrm{u}_{\mathrm{disc}}\right|\right.$ $\left.\left(\mathrm{u}_{\text {ball }}+\mathrm{u}_{\text {disc }}\right) / 2\right] \times 100 \%$. Thus, $\mathrm{SRR}=0 \%$ (i.e., $\mathrm{u}_{\text {ball }}=$ $\mathrm{u}_{\mathrm{disk}}$ ) represents a pure rolling contact and higher SRR 
values represent a higher portion of the sliding character. With the software provided by the manufacturer (PCS Instruments, MTM version 1.0, London, UK), values in the range of SRR 1-200\% are accessible. For this study, an SRR value of $10 \%$ was chosen, which enables friction measurements in conditions close to pure rolling. Speeddependence tests on polymer solutions $(0.25$ or $0.5 \mathrm{mg} \mathrm{mL}^{-1}$ depending on molecular weight) were carried out at a constant load of $10 \mathrm{~N}$ (Hertzian contact pressure, $0.42 \mathrm{GPa}$ ) and at a controlled temperature $\left(25^{\circ} \mathrm{C}\right)$. The disk track radius was fixed at $21 \mathrm{~mm}$, and for each measurement a new ball and a new disk were used. Balls and disks were cleaned according to the same procedure used for pin-on-disk tribometry experiments, except that air-plasma was used instead of oxygen-plasma. The instrumental parts expected to be in contact with the polymer solution were cleaned with a commercial cleaner (Hydrochloric acid $300 \mathrm{mmol} / \mathrm{L}$ and detergent 1\%, Roche Diagnostics GmbH, Mannheim, Germany).

\section{Results and Discussion}

\subsection{Synthesis and Structural Features of PLL-g-dex Copolymers}

PLL-g-dextran copolymers with different molecular weights and grafting ratios of dextran side chains were successfully synthesized and characterized by ${ }^{1} \mathrm{H}-\mathrm{NMR}$. The molecular weights of the selected dextrans were 5.2 and $10 \mathrm{kDa}$ (denoted as $\operatorname{dex}(5.2)$ and $\operatorname{dex}(10))$. Grafting ratio was varied between roughly 3 and 9 and evaluated by means of NMR and EA. As a reference, a series of PLL (20)-g-PEG(5) (20 kDa for the molecular weight of PLL backbone and $5 \mathrm{kDa}$ for the molecular weight of PEG side chains) with varying grafting ratios, roughly $g$ [3] to $g$ [11], were purchased from SuSoS AG (Dübendorf, Switzerland). In Table 1, the structural features of the synthesized copolymers and the yield of the synthesis are presented in detail.

It should be noted that the two dextran polymers with different molecular weights in this study, $\operatorname{dex}(5.2)$ and $\operatorname{dex}(10)$, were selected to maintain the structural features that may critically influence adsorption and lubricating properties comparable with those of PLL(20)- $g$-PEG(5). Firstly, $\operatorname{dex}(5.2)$ is nearly identical with $\operatorname{PEG}(5)$ in molecular weight, and thus can be employed to generate PLL-g-dex copolymers with similar molecular weights to those of PLL- $g$-PEG at the same grafting ratio. As will be addressed below, the molecular weights of the copolymers need to be comparable when the diffusion properties of copolymers through the liquid medium play an important role in the lubricating behavior.

PEG(5) is composed of 113.6 monomer units (ethylene glycol (EG)), whose fully extended chain length is estimated to be $40.5 \mathrm{~nm}$ (based on the molecular length of EG, $0.358 \mathrm{~nm}$ [46]), whereas $\operatorname{dex}(5.2)$ is composed of 31.8 monomer units (sugar rings), whose fully extended chain length is estimated to be only $22.3 \mathrm{~nm}$ (based on the molecular length of dextran: $0.7 \mathrm{~nm}$ [47]). While the lubricating film thickness generated by the adsorption of comb-like copolymer will be determined by many

Table 1 Synthesized PLL-g-dex copolymers and PLL-g-PEG copolymers used for comparison purposes

\begin{tabular}{|c|c|c|c|c|c|}
\hline Polymer & $\begin{array}{l}\text { Synthesis } \\
\text { yield [\%] }\end{array}$ & $\begin{array}{l}\text { No. of grafted } \\
\text { side chains } \\
\text { per PLL }\end{array}$ & $\begin{array}{l}\text { No. of free } \\
\text { lysines per PLL }\end{array}$ & $\begin{array}{l}\text { Percentage of } \\
\text { side-chain } \\
\text { grafting }(\%)\end{array}$ & $\begin{array}{l}\text { M.W. of } \\
\text { copolymer (kDa) }\end{array}$ \\
\hline PLL(20)-g[3.4]-dex(5.2) & 30 & 28.15 & 67.5 & 29.4 & 157.4 \\
\hline PLL(20)-g[5.3]-dex(5.2) & 60 & 18.06 & 77.6 & 18.9 & 105.4 \\
\hline PLL(20)-g[6.6]-dex(5.2) & 56 & 14.50 & 81.2 & 15.2 & 87.0 \\
\hline PLL(20)-g[7.3]-dex(5.2) & 27 & 13.11 & 82.6 & 13.7 & 80.0 \\
\hline PLL(20)-g[8.7]-dex(5.2) & 60 & 11.00 & 84.7 & 11.5 & 69.0 \\
\hline PLL(20)-g[3.7]-dex(10) & 46 & 25.86 & 69.8 & 27.0 & 270.1 \\
\hline PLL(20)-g[4.8]-dex(10) & 40 & 19.94 & 75.8 & 20.8 & 211.6 \\
\hline PLL(20)-g[6.5]-dex(10) & 59 & 14.72 & 81.0 & 15.4 & 159.5 \\
\hline PLL(20)-g[8.6]-dex(10) & 58 & 11.13 & 84.6 & 11.6 & 123.5 \\
\hline PLL(20)-g[3]-PEG(5) & - & 31.90 & 63.8 & 33.3 & 171.8 \\
\hline PLL(20)-g[4.4]-PEG(5) & - & 21.75 & 73.9 & 22.7 & 121.0 \\
\hline PLL(20)-g[6.6]-PEG(5) & - & 14.50 & 81.2 & 15.2 & 84.8 \\
\hline PLL(20)-g[11.2]-PEG(5) & - & 8.54 & 87.1 & 8.9 & 55.0 \\
\hline
\end{tabular}

The numbers of lysine units for the PLL-HBr $(20 \mathrm{kDa})$, before grafting with dextran or PEG, is 95.7. The numbers of monomer units for the PEG $(5 \mathrm{kDa})$ and dex $(5.2 \mathrm{kDa})$ are 113.6 and 31.8 , respectively 
parameters, the fully extended length of side chains will set the upper bound of the film thickness. For this reason, $\operatorname{dex}(10)$ (fully extended length, $44.6 \mathrm{~nm}$ ) was employed to generate PLL- $g$-dex copolymers that can potentially generate comparable film thicknesses with those of PLL-g-PEG(5) copolymers. The molecular weights of PLL- $g$-dex(10) are, of course, significantly higher than those of PLL- $g$-dex(5.2) or PLL- $g$-PEG(5) for the same grafting ratio (see Table 1).

\subsection{Adsorption Behavior of PLL-g-dex: OWLS}

A representative adsorption profile for PLL- $g$-dex copolymer onto the oxide surface as characterized by OWLS is presented in Fig. 2 (for the case of PLL(20)-g[3.4]-dex(5.2)). Upon exposure of a waveguide surface to the polymer solution (after ca. $60 \mathrm{~min}$ exposure to HEPES buffer to achieve the baseline), the adsorption of PLL-g-dex proceeded very rapidly such that more than the $90 \%$ of the final mass of adsorbed polymer was realized within the first $5 \mathrm{~min}$. Because the raw data signals (change in the refractive index) at this stage reflect the contributions from both the surface-bound polymers and the bulk polymer solution, the final adsorbed mass was determined after the flow cell was rinsed with buffer solution (after ca. $50 \mathrm{~min}$ exposure to the polymer solution) to exclude the contribution from the bulk solution and loosely bound polymers. The negligible reduction in the adsorbed mass after the rinse indicates that no noticeable polymer desorption occurred during rinsing and assures the formation of a stable polymer adlayer. The same procedure was repeated for all the PLL- $g$-dex and PLL- $g$-PEG copolymers, as well as $\operatorname{dex}(5.2)$. The resulting adsorbed masses per unit area are presented as a function of grafting ratio for the series of PLL- $g$-dex(5.2), PLL-g-dex(10), and PLL- $g$-PEG(5) copolymers, as shown in Fig. 3.

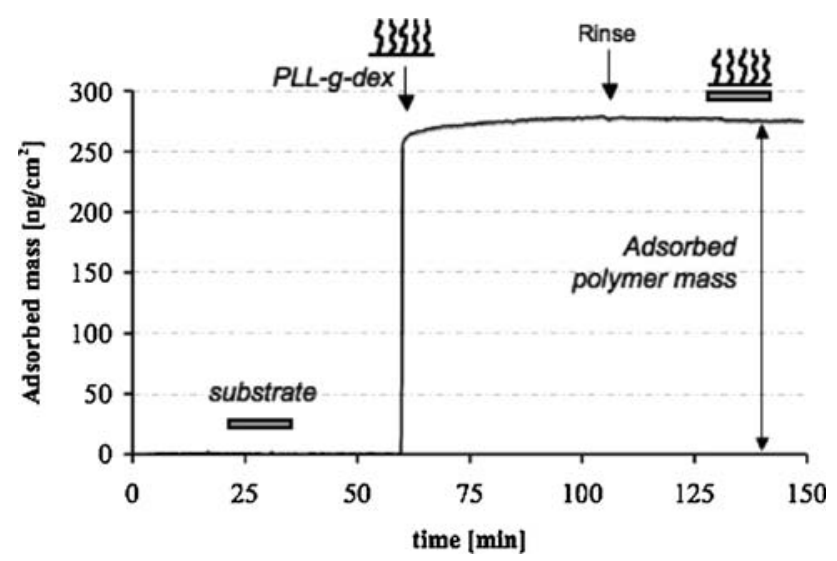

Fig. 2 In situ OWLS measurement of the polymer adsorption from solution onto uncoated waveguide chips

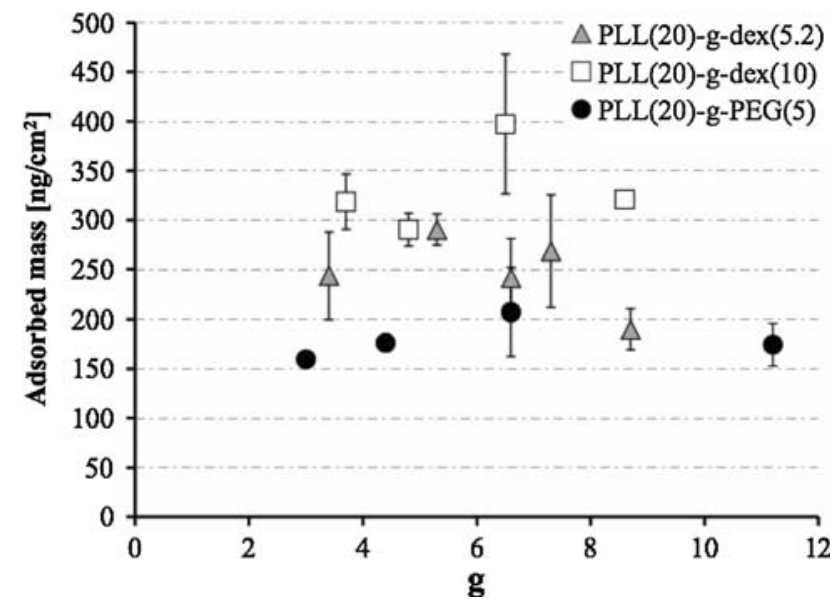

Fig. 3 OWLS results for the adsorption of PLL- $g$-dex copolymers and the counterpart PLL- $g$-PEG copolymers employed in this work. For some data points, error bars are smaller than the symbols

All the copolymers employed in this study showed a significant amount of adsorption onto the OWLS waveguide surfaces, ranging from ca. 200 to $290 \mathrm{ng} \mathrm{cm}^{-2}$ for PLL-g-dex(5.2), ca. 290 to $390 \mathrm{ng} \mathrm{cm}^{-2}$ for PLL-g-dex(10), and ca. 160 to $200 \mathrm{ng} \mathrm{cm}^{-2}$ for PLL- $g$-PEG(5) on average (see Table 2 and Fig. 3). Meanwhile, dextran $(\operatorname{dex}(5.2))$ alone revealed negligible adsorption onto the surfaces $\left(5.6 \pm 3.7 \mathrm{ng} \mathrm{cm}^{-2}\right)$. This observation supports the hypothesis that both the adsorption mechanism and the conformation of the PLL- $g$-dex copolymer on negatively charged surfaces are very similar to those of PLL- $g$-PEG copolymer [5, 6, 9]; the adsorption of the copolymer proceeds through an electrostatic interaction between the PLL backbone and the surface in an aqueous environment, and thus the PLL backbone generally lies flat on the substrate surface, whereas the dextran side chains stretch into bulk aqueous solution because of their hydrophilic characteristics, forming brush layers. As shown in Fig. 3, the PLL-g-dex(10) copolymers employed in this study lead to either comparable or slightly higher adsorbed masses compared with PLL(20)-g-dex(5.2) copolymers, whereas both of them show somewhat higher adsorbed masses compared to PLL(20)-g-PEG(5) copolymers. In addition, for all three groups of copolymers reveal that the adsorbed mass is not significantly influenced by the variation in grafting ratio within the range selected in this study. The adsorbed mass is the product of the molecular weight of each copolymer molecule and the number of moles adsorbed on the surface. Because the (average) molecular weights of the copolymers are precisely known from their structures, more insights into the adsorption behavior of the copolymers can be obtained by decomposing their adsorbed mass into various coverage parameters, as shown in Fig. 4.

First, the molecular weights of the copolymers are shown as a function of the inverse of grafting ratio, $1 / \mathrm{g}$, in 
Table 2 Summary of the adsorption data determined by OWLS for the synthesized PLL- $g$-dex copolymers

\begin{tabular}{lllllll}
\hline Surface & $m_{\text {pol }}\left[\mathrm{ng} / \mathrm{cm}^{2}\right]$ & $n_{\text {Lys }}\left[1 / \mathrm{nm}^{2}\right]$ & $n_{\text {dex or PEG }\left[1 / \mathrm{nm}^{2}\right]}$ & $n_{\text {monomer units dex or PEG }\left[1 / \mathrm{nm}^{2}\right]} \mathrm{L}[\mathrm{nm}]$ & $\mathrm{L} / 2 \mathrm{Rg}$ \\
\hline PLL(20)-g[3.4]-dex(5.2) & $244 \pm 44$ & $0.89 \pm 0.20$ & $0.26 \pm 0.05$ & $8.36 \pm 1.51$ & $2.12 \pm 0.21$ & $0.45 \pm 0.04$ \\
PLL(20)-g[5.3]-dex(5.2) & $290 \pm 16$ & $1.59 \pm 0.09$ & $0.30 \pm 0.02$ & $9.53 \pm 0.52$ & $1.96 \pm 0.05$ & $0.42 \pm 0.01$ \\
PLL(20)-g[6.6]-dex(5.2) & $241 \pm 40$ & $1.60 \pm 0.26$ & $0.24 \pm 0.04$ & $7.71 \pm 1.27$ & $2.19 \pm 0.18$ & $0.47 \pm 0.04$ \\
PLL(20)-g[7.3]-dex(5.2) & $269 \pm 57$ & $1.94 \pm 0.41$ & $0.27 \pm 0.06$ & $8.45 \pm 1.80$ & $2.10 \pm 0.22$ & $0.45 \pm 0.05$ \\
PLL(20)-g[8.7]-dex(5.2) & $190 \pm 21$ & $1.59 \pm 0.17$ & $0.18 \pm 0.02$ & $5.80 \pm 0.62$ & $2.52 \pm 0.1$ & $0.54 \pm 0.03$ \\
PLL(20)-g[3.7]-dex(10) & $319 \pm 28$ & $0.68 \pm 0.06$ & $0.18 \pm 0.02$ & $10.16 \pm 0.58$ & $2.51 \pm 0.11$ & $0.36 \pm 0.02$ \\
PLL(20)-g[4.8]-dex(10) & $290 \pm 17$ & $0.79 \pm 0.05$ & $0.16 \pm 0.01$ & $10.16 \pm 0.49$ & $2.65 \pm 0.08$ & $0.38 \pm 0.01$ \\
PLL(20)-g[6.5]-dex(10) & $394 \pm 100$ & $1.43 \pm 0.36$ & $0.22 \pm 0.06$ & $13.53 \pm 3.43$ & $2.32 \pm 0.30$ & $0.33 \pm 0.04$ \\
PLL(20)-g[8.6]-dex(10) & $321 \pm 2$ & $1.50 \pm 0.01$ & $0.17 \pm 0.00$ & $10.75 \pm 0.07$ & $2.57 \pm 0.01$ & $0.37 \pm 0.00$ \\
PLL(20)-g(3)-PEG(5) & $160 \pm 3$ & $0.54 \pm 0.00$ & $0.18 \pm 0.00$ & $20.49 \pm 0.07$ & $2.53 \pm 0.00$ & $0.45 \pm 0.00$ \\
PLL(20)-g[4.4]-PEG(5) & $180 \pm 8$ & $0.86 \pm 0.04$ & $0.19 \pm 0.01$ & $22.14 \pm 1.04$ & $2.44 \pm 0.06$ & $0.43 \pm 0.01$ \\
PLL(20)-g[6.6]-PEG(5) & $218 \pm 58$ & $1.48 \pm 0.39$ & $0.22 \pm 0.06$ & $25.47 \pm 6.71$ & $2.30 \pm 0.31$ & $0.41 \pm 0.05$ \\
PLL(20)-g[11.2]-PEG(5) & $184 \pm 20$ & $1.92 \pm 0.20$ & $0.17 \pm 0.02$ & $19.52 \pm 2.03$ & $2.60 \pm 0.14$ & $0.46 \pm 0.02$ \\
\hline
\end{tabular}

$m_{\text {pol }}$ adsorbed polymer mass, $m_{\text {serum }}$ mass of serum adsorbed, $n_{\text {lys }}$ surface density of lysine monomers, $n_{\text {dex }}$ or $P E G$ surface density of dextran or

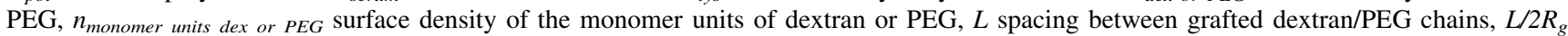
degree of overlap of dextran/PEG chains

Fig. 4 Molecular weight (a), lysine monomer density $\left(n_{\text {lys }}\right)$ (b), dextran $\left(n_{\mathrm{dex}}\right)$ or PEG $\left(n_{\mathrm{PEG}}\right)$ chain density $(\mathbf{c})$, and density of the monomer units of dextran $\left(n_{\text {monomer units dex }}\right)$ or PEG ( $\left.\mathrm{n}_{\text {monomer units PEG }}\right)(\mathbf{d})$ of the synthesized PLL-g-dex copolymers and the counterpart PLL- $g$-PEG copolymers on the silica-titania surface. For some data points, error bars are smaller than the symbols
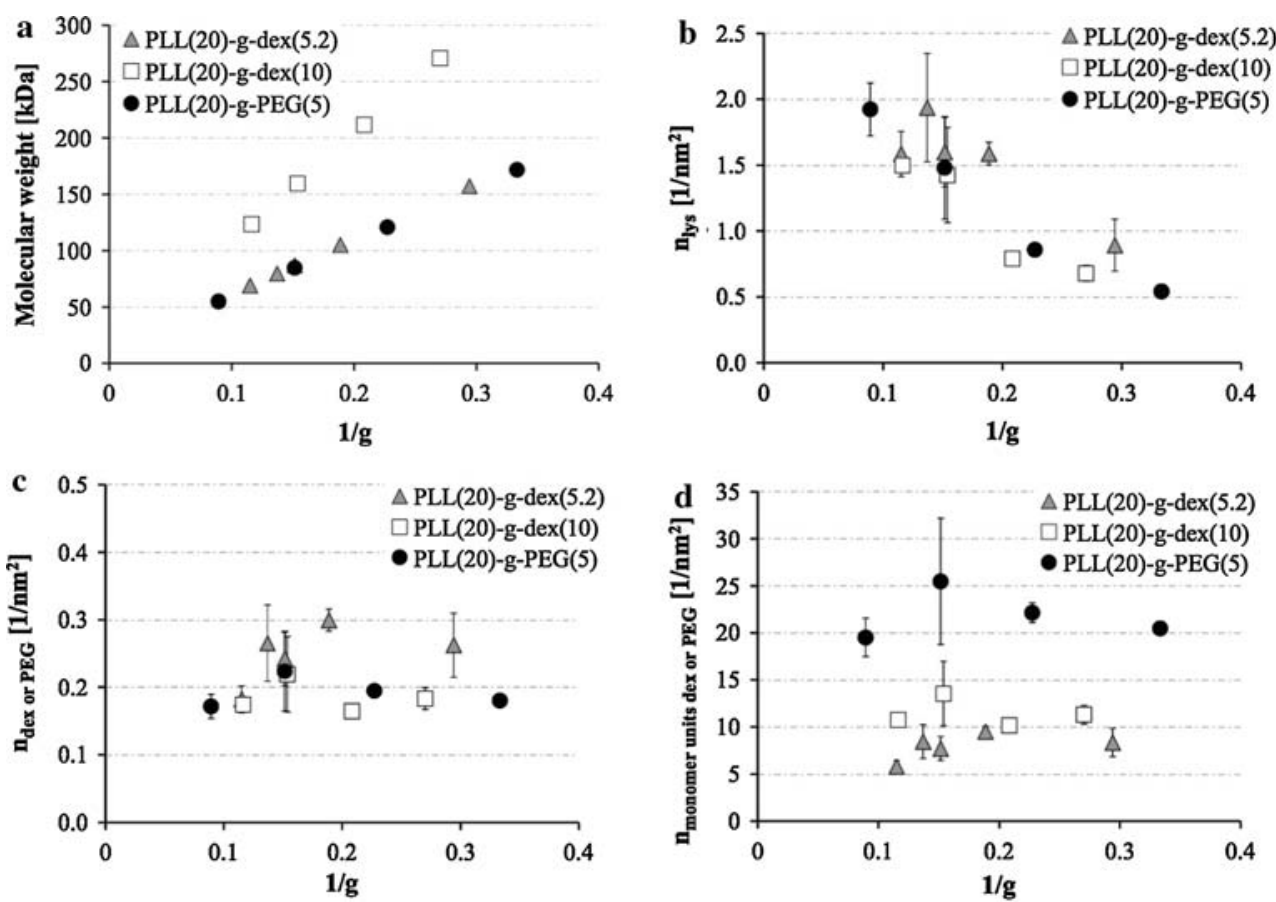

Fig. 4(a); in this plot, increasing the value of $1 / g$ represents the increase in the number of grafted side chains along the PLL backbone. In general, the molecular weight of the copolymer increases linearly with increasing $1 / g$ values, due to increasing number of grafted side chains. The variation of molecular weight as a function of grafting ratio is nearly identical for PLL- $g$-PEG(5) and PLL- $g$-dex(5.2), while the molecular weights of PLL- $g$-dex(10) copolymers are much higher. In turn, from the adsorbed mass and the compositional features of the copolymers, it is possible to calculate the surface concentration of lysine monomers, $n_{\text {lys }}$, and dextran or PEG chains, $n_{\mathrm{dex}}$ or PEG. The surface concentration of lysine monomers, $n_{\text {lys }}$, reflects the number of copolymer molecules on the surface, whereas the surface density of dextran or PEG chains reflects the efficacy of the copolymer in grafting the hydrophilic polymer chains (either dextran or PEG) onto the surface. For the case of dextrans, because two different molecular weights (dextran chain lengths) were employed to generate PLL-g-dex copolymers in this study, the efficacy of grafting hydrophilic moieties can be expressed by the surface concentration of dextran monomer units (sugar rings) or 


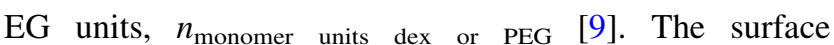
concentration of lysine monomers, $n_{\text {Lys }}$, dextran or PEG chains, $n_{\mathrm{dex}}$ or $n_{\mathrm{PEG}}$, and monomer units of them, $n_{\text {monomer }}$

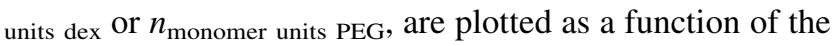
inverse of grafting ratio, $1 / g$, in Fig. $4 b$, c, and d, respectively. Finally, the mean spacing between the dextran chains, $L$, as well as the ratio between the spacing and the radius of gyration of dextran chains, $L / 2 R_{g}$, can be obtained to estimate the conformation of the surface-grafted dextran or PEG chains [9]. The results of these calculations are summarized in Table 2.

As shown in Fig. 4b, the surface concentrations of lysine monomers, $n_{\text {Lys }}$, for both PLL(20)- $g$-dex(5.2) copolymers show roughly constant values until $1 / g$ reaches ca. 0.2 , and yet rapidly decrease at higher $1 / g$ values. Degraded adsorption with increasing number of side chains (increasing $1 / g$ ) may be due either to the decreasing number of anchoring units (free $\mathrm{NH}_{3}{ }^{+}$) and/or increasing steric hindrance between grafted side chains. A previous study [9] involving PLL(20)- $g$-PEG and varying the molecular weight of PEG side chains, including PEG(1), PEG(2), and PEG(5), has, however, confirmed that this trend is mainly driven by the steric hindrance between side chains; a decrease in $n_{\text {Lys }}$ with increasing $1 / g$ was observed in the cases of PLL(20)- $g$-PEG(2) and PLL(20)-g-PEG(5), but not in the case of PLL- $g$-PEG(1), which has smaller steric hindrance between side chains as a consequence of the PEG(1)'s smaller radius of gyration. For PLL(20)-g-dex(10) and PLL(20)- $g$-PEG(5), a linear decrease of $n_{\text {Lys }}$ is observed from $1 / g \approx 0.1$ to the highest value of $\approx 0.28$, which implies that the steric hindrance between $\operatorname{dex}(10)$ is more serious than in the other two cases. The radii of gyration for the three hydrophilic polymers are in the order $\operatorname{dex}(5.2)(2.35 \mathrm{~nm}[48])<\operatorname{PEG}(5)$ (2.82 nm [49]) $<\operatorname{dex}(10)$ (3.49 $\mathrm{nm}[48])$.

The surface density of dextran or PEG chains, Fig. 4c, is thus proportional to the product of the $n_{\text {Lys }}$ and the number of side chains per molecule, which also linearly increases with increasing $1 / g$ values (see the Table 1). For PLL(20)-g-dex(5.2) copolymers, the increase in number of grafted side chains on the backbone leads to a proportional increase in the grafted side chains on the surface in the initial $1 / g$ range of $0.1 \sim 0.2$, and yet, $n_{\mathrm{dex}}$ or $n_{\mathrm{PEG}}$ reaches a plateau at higher $1 / g$ values. Apparently, this plateau is a result of opposing effects with increasing $1 / g$ - the decreasing number of copolymers on the surface, as shown by the decrease of $n_{\text {Lys }}$, and increasing number of grafted side chain on the backbone. The radii of gyration for $\operatorname{dex}(10)$ and PEG(5) are larger than that of $\operatorname{dex}(5)$, and the plateau for the corresponding copolymers is already reached at $1 / g=0.1$. Finally, the monomer densities, as shown in Fig. 4d, reveal nearly the same trends with those of $n_{\mathrm{dex}}$ or $n_{\mathrm{PEG}}$, the relative magnitudes between $\operatorname{dex}(5.2)$ and $\operatorname{dex}(10)$ being reversed.
3.3 Lubrication Properties of the Copolymers: General Considerations

To evaluate the lubricating properties of PLL- $g$-dex copolymer solutions and compare them with other standard aqueous solutions, including HEPES buffer solution, dextran solutions (dex(5.2)), as well as PLL(20)- $g$-PEG(5) solutions, coefficient of friction $(\mu)$ versus speed plots were acquired under both sliding and mixed sliding/rolling conditions using a pin-on-disk tribometer and MTM, respectively.

The lubricating capabilities of the copolymers in this study are considered to be directly associated with their adsorption properties onto the tribopair surfaces. Although the adsorption properties of the copolymers onto the pins (stainless steel) remain uncharacterized, the OWLS measurements shown in the previous section can represent the adsorption properties of the copolymers on the disk (glass) because silicon oxides prevail on both the waveguide and the glass disk surfaces. Given that not only adsorbed masses but also lysine monomer densities, $n_{\text {Lys }}$, hydrophilic polymer chain densities, $n_{\mathrm{dex}}$ or $n_{\mathrm{PEG}}$, as well as the hydrophilic monomer unit densities, $n_{\text {monomer units dex }}$ or

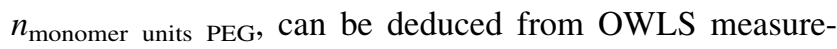
ments (Table 2 and Fig. 4), it is of interest to see with which structural parameter the lubricating properties are best correlated.

In macroscopic sliding contacts between two rigid surfaces, as in this study, the lubricating capabilities of the copolymers are expected to be further influenced by their adsorption kinetics. As was previously addressed in a study employing fluorescently labeled PLL- $g$-PEG copolymers, [50] the electrostatically adsorbed polymer layers are easily scraped away from the surfaces during sliding contacts in pin-on-disk tribometry, particularly due to asperity contacts arising from surface roughness and consequently high local contact pressures. Nevertheless, the excess polymers in bulk solution, if present, re-adsorb onto the area where the initially adsorbed copolymers have been desorbed, and the tribostressed area 'self-heals'. In other words, effective lubrication of electrostatically adsorbed copolymers over a long period in the presence of excess polymer in bulk solution is rather due to their fast adsorption kinetics than their adsorption strength. An important prerequisite to test the efficacy of the copolymers as lubricant additives and to take into account their surface adsorption kinetics behavior is to keep the number of excess polymer molecules constant, i.e., their molar concentration available to 'heal' the tribostressed area. For this reason, we have doubled the mass concentration of PLL(20)-g-dex(10) copolymers, $0.5 \mathrm{mg} / \mathrm{mL}$, with respect to those of PLL(20)- $g$-dex(5.2) and PLL(20)- $g$-PEG(5) copolymers, $0.25 \mathrm{mg} / \mathrm{mL}$, for the tribological tests. If the mass concentrations were kept constant for all the copolymers, the molar concentrations of 


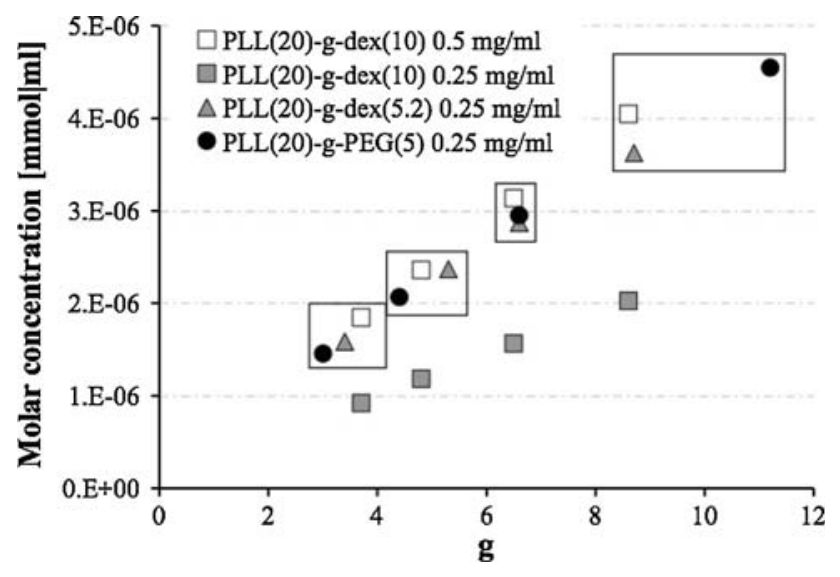

Fig. 5 Molar concentration $[\mathrm{mmol} / \mathrm{ml}]$ of the copolymer solutions employed in this study (highlighted in the squares the concentrations chosen for the characterization of the lubricating properties of PLL- $g$-dex and the counterpart PLL- $g$-PEG)

PLL-g-dex(10) copolymers would be significantly lower than those of the other two types of copolymers, as shown in Fig. 5.

\subsubsection{Lubrication at Sliding Contacts: Pin-on-disk Tribometry}

Sliding-contact experiments, carried out by pin-on-disk tribometry, were intended to assess the lubricating capabilities of the polymer solutions in the boundarylubrication regime. To compare the relative lubricating properties of three kinds of copolymers, PLL(20)- $g$ dex(5.2), PLL(20)-g-PEG(5), and PLL(20)-g-dex(10), they were grouped mainly according to grafting ratio ( $x$-axis) as shown in Fig. 5. As mentioned above, this was to keep the molar concentration ( $y$-axis) nearly constant for the copolymers employed for the comparison. As shown in Fig. 6, all the copolymer solutions employed in this study

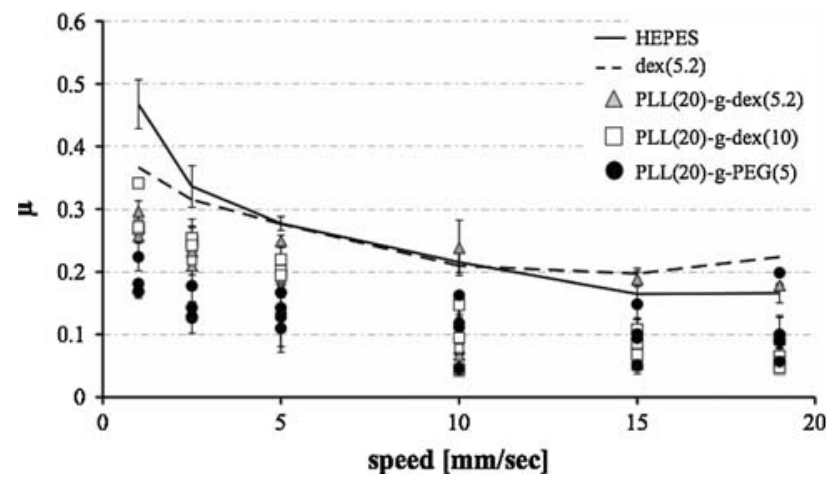

Fig. 6 Sliding pin-on-disk results of all PLL- $g$-dex and PLL- $g$-PEG copolymers (with grafting ratio varying from 3 to 11 ) investigated in this study (tribopair: steel balls versus glass disks, load: $2 \mathrm{~N}$, room temperature): coefficients of friction $(\mu)$ as a function of the sliding speed led to apparent reduction in $\mu$ values compared to those of HEPES buffer solution (as much as $50 \%$ for most copolymers at speeds above $10 \mathrm{~mm} \mathrm{~s}^{-1}$, generally ca. $30 \%$ reduction at lower speeds). In contrast, the dextran (5.2) solution revealed negligible improvement in the lubricating properties. This behavior is consistent with the very low adsorption of dex(5.2) onto the oxide surfaces, as determined by OWLS measurements (Table 2). To highlight the comparison of the lubricating properties of PLL- $g$-PEG and PLL- $g$-dex, however, the data for the HEPES buffer solutions and $\operatorname{dex}(5.2)$ are omitted in subsequent figures.

The $\mu$ vs. speed plots for the copolymers with $g$ [3.5], $g$ [4.8], $g$ [6.5], and high $g[y]$, between ca. $g$ [8] and $g$ [11] (average values) are plotted in Figs. 7, 8, 9, and 10, respectively. Generally, $\mu$ values $(\operatorname{load}=2 \mathrm{~N}$ ) of the

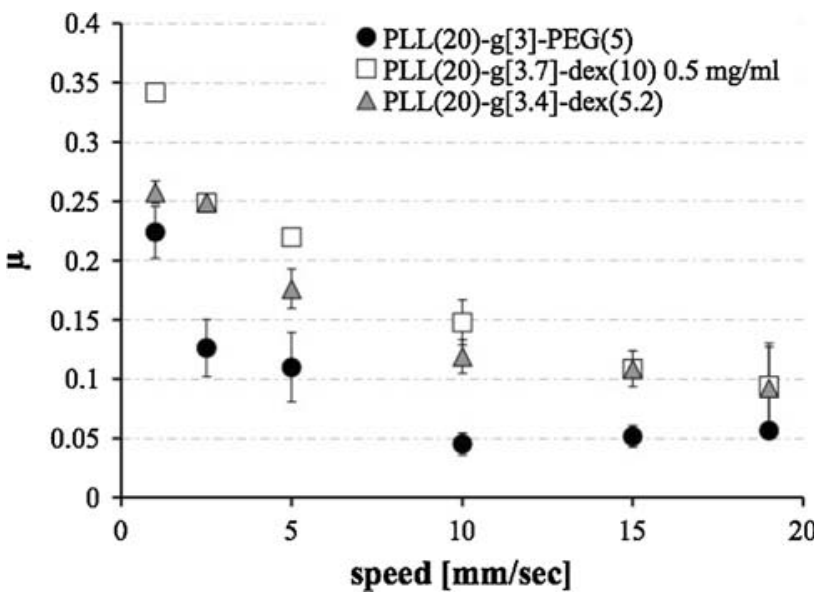

Fig. 7 Sliding pin-on-disk results of the PLL- $g$-dex copolymers and PLL- $g$-PEG counterpart with ca. $g$ [3.5]: coefficients of friction as a function of the sliding speed. For some data points, error bars are smaller than the symbols

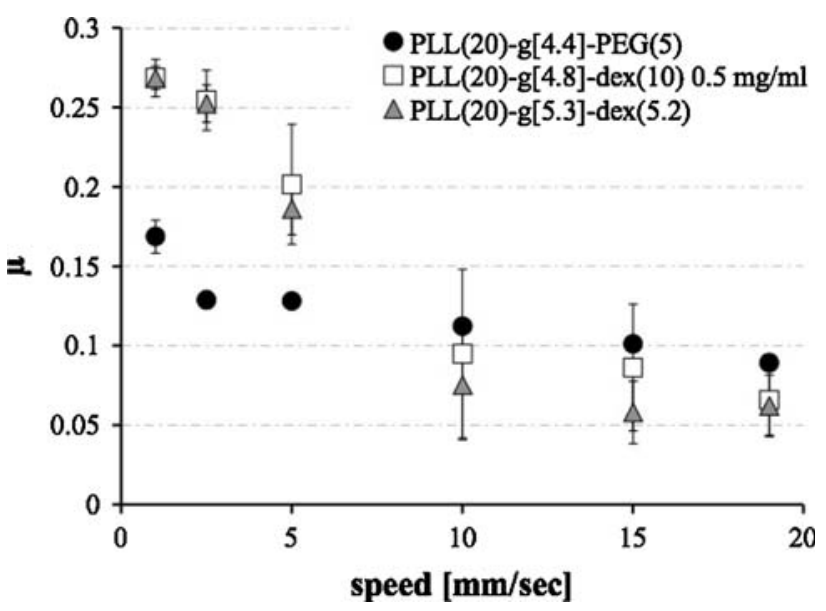

Fig. 8 Sliding pin-on-disk results of the PLL- $g$-dex copolymers and PLL- $g$-PEG counterpart with ca. $g$ [4.8]: coefficients of friction as a function of the sliding speed. For some data points, error bars are smaller than the symbols 


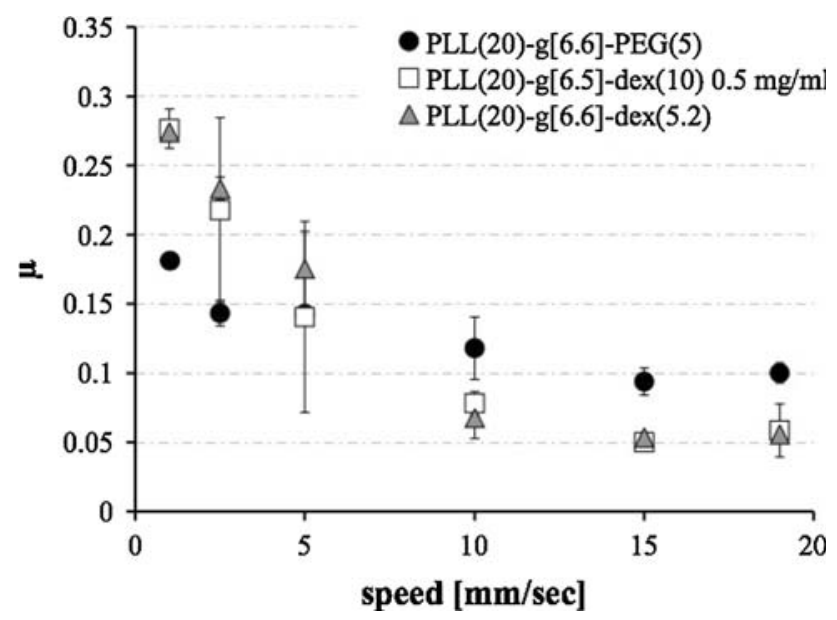

Fig. 9 Sliding pin-on-disk results of the PLL-g-dex copolymers and PLL- $g$-PEG counterpart with ca. $g$ [6.5]: coefficients of friction as a function of the sliding speed. For some data points, error bars are smaller than the symbols

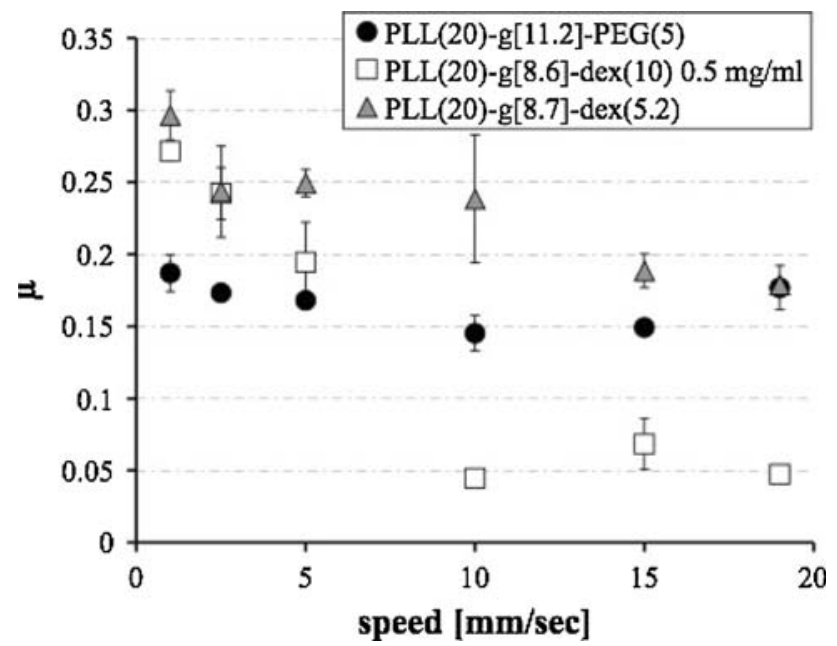

Fig. 10 Sliding pin-on-disk results of the PLL- $g$-dex copolymers and PLL- $g$-PEG counterpart with high grafting ratio, between ca. $g[8]$ and $g$ [11]: coefficients of friction as a function of the sliding speed. For some data points, error bars are smaller than the symbols

copolymer solutions revealed an initial reduction with increasing sliding speed (corresponding to increasing frequency of tribocontact), but they started to level off from approximately $10 \mathrm{~mm} \mathrm{~s}^{-1}$ to the highest speed investigated, reaching approximately $\mu=0.05$ for the bestbehaving copolymer in each comparison set. As shown in Fig. 7, for the copolymers possessing the grafting ratio of $\sim g$ [3.5], the $\mu$ values of the two PLL-g-dex solutions were observed to be generally very similar, whereas those of PLL(20)-g-PEG(5) are slightly, yet noticeably, lower than the two PLL-g-dex solutions over the entire speed regime. For all other cases, as shown in Figs. 8, 9, and 10, the relative lubricating efficacy of PLL-g-dex copolymers compared with PLL(20)-g-PEG(5) was highly dependent upon the sliding speed; in the low-speed regime $\left(\leq 10 \mathrm{~mm} \mathrm{~s}^{-1}\right)$, the lubricating performance of PLL(20)- $g$ PEG(5) was consistently superior to those of PLL- $g$-dex copolymers. In the high-speed regime $\left(\geq 10 \mathrm{~mm} \mathrm{~s}^{-1}\right)$, however, PLL-g-dex copolymers reveal lower friction forces than PLL(20)-g-PEG(5) in most cases. Exceptions are the cases of $\sim g[3.5]$ (Fig. 7) and PLL(20)- $g$ [8.6]$\operatorname{dex}(5.2)$ (Fig. 10), which have too high a grafting ratio to be in a brush conformation.

To more clearly visualize the relative magnitude of lubricating efficacy of the copolymers employed, and also to visualize the variation of the lubricating properties as a function of architectural features of the copolymers, the $\mu$ values representing the characteristics in the low-speed regime $\left(1 \mathrm{~mm} \mathrm{~s}^{-1}\right)$ and the high-speed regime $\left(19 \mathrm{~mm} \mathrm{~s}^{-1}\right)$ are plotted against the inverse of grafting ratio, $1 / g$, in Figs. 11 and 12, respectively. It should be borne in mind that all speeds investigated in the pin-ondisk studies correspond to the boundary lubrication regime, and that the frequency of tribocontact is the parameter that is being investigated via speed changes.

In the low-speed regime, (Fig. 11), the $\mu$ values of PLL(20)- $g$-PEG(5) compared with PLL- $g$-dex copolymers are, again, consistently lower over the entire range of grafting ratio. Because the magnitudes of extension of the PEG and dextran chains, as quantified by $L / 2 R_{\mathrm{g}}$, are comparable for the PLL(20)-g-PEG(5) and PLL(20)-g-dex(5.2) copolymers (see the Table 2), and in fact, those of PLL(20)-g-dex(10) are even greater (lower values of $L / 2 R_{\mathrm{g}}$ ) than PLL(20)- $g$-PEG(5), it is unlikely that the difference in the conformation of the two different hydrophilic polymer chains on the surface is the principal cause of the

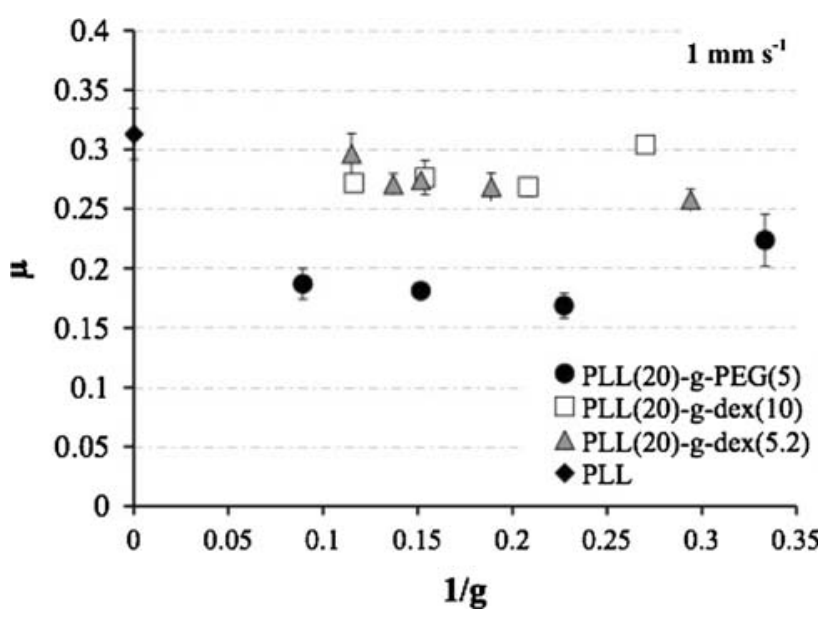

Fig. 11 Sliding pin-on-disk results of all PLL- $g$-dex and PLL- $g$-PEG copolymers investigated in this study at the lowest speed investigated $\left(1 \mathrm{~mm} \mathrm{~s}^{-1}\right)$ : coefficients of friction as a function of the degree of grafting of the side chains, $1 / g$. For some data points, error bars are smaller than the symbols 


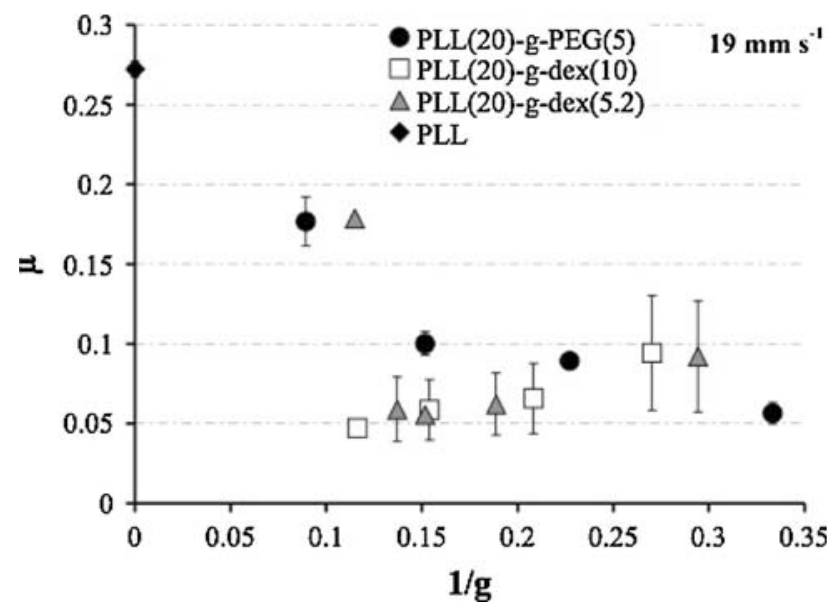

Fig. 12 Sliding pin-on-disk results of all PLL- $g$-dex and PLL- $g$-PEG copolymers investigated in this study at the highest speed investigated $\left(19 \mathrm{~mm} \mathrm{~s}^{-1}\right)$ : coefficients of friction as a function of the degree of grafting of the side chains, $1 / \mathrm{g}$. For some data points, error bars are smaller than the symbols

superior lubricating properties of PLL(20)- $g$-PEG(5) under these conditions. Furthermore, based upon $L / 2 R_{\mathrm{g}}$ values and the fully extended lengths of PEG(5) and $\operatorname{dex}(10)$, the film thicknesses are also estimated to be comparable for the two hydrophilic polymer chains. We thus speculate that dextran and PEG chains may display different innate water-based lubricating capabilities, mainly arising from different degrees and mechanisms of hydration and/or differences in the stiffness and/or dynamical behavior of the chains [51]. Quantitative measurements of the hydration for PLL-g-PEG and PLL-g-dex copolymers are currently in progress.

From Fig. 11, it is also noticeable that in the low-speed regime, the $\mu$ values of all the copolymers remain nearly constant over the entire range of grafting ratio, except for the onset of an increase in $\mu$ for PLL- $g$-PEG(5) and PLL- $g$-dex(10) at the highest $1 / g$ value, i.e., the highest number of grafted side chains along the PLL backbone. It is important to note that although the molar concentrations of the copolymers were controlled to be constant for each set of three copolymers with similar grafting ratio, the molar concentrations of the copolymers do, of course, increase with increasing grafting ratio, (i.e., decrease with increasing $1 / g$ ) as shown in Fig. 4a. Thus, the molar concentrations of the copolymers shown in Fig. 11 are different as much as a factor of three. In turn, generally constant $\mu$ values observed over the entire $1 / g$ range in Fig. 11 suggest that the lubricating properties in the lowspeed regime are not sensitively influenced by the variation of the molar concentration. As will be discussed in more detail below, this feature is in stark contrast to the lubricating behavior in the high-speed regime, and indicates that the lubricating properties of the copolymers in the low-speed regime are mainly governed by the adsorption properties in equilibrium, since the solution concentration is considerably in excess of that necessary to achieve monolayer coverage on the sliding surfaces.

Comparing the various parameters representing adsorption properties of the copolymers, including adsorbed mass, the surface densities of lysine units, $n_{\text {Lys }}$, surface densities of dextran or PEG chains, $n_{\mathrm{dex}}$ or $n_{\mathrm{PEG}}$, surface densities of monomer units, $n_{\text {monomer units dex }}$ or $n_{\text {monomer units PEG }}$, and the magnitude of brush extension, $L / 2 R_{\mathrm{g}}$ (Table 2 and Fig. 4), the lubricating properties appear to be most highly correlated with the surface densities of dextran or PEG chains, albeit not in a simple, linear fashion; both lubricating properties and surface densities were usually insensitive to the variation of grafting ratio within the selected range. The onset of the increase in $\mu$ values observed from PLL- $g$-PEG(5) and PLL- $g$-dex(10), but not from PLL- $g$-dex(5.2), is associated with the radius of gyration for the side chains changing in the order of $\operatorname{dex}(5.2)<\operatorname{PEG}(5)<\operatorname{dex}(10)$. As was previously stated, too high a number of the grafted side chains along the PLL backbone tends to degrade the adsorption properties of comb-like graft copolymers, and consequently their lubricating properties. Because the steric hindrance between neighboring side chains plays a major role in this trend, larger radii of gyration of the side chains are expected to exacerbate such behavior.

In the high-speed regime, (Fig. 12), $\mu$ values of all copolymers employed in this work fall in the same range, between 0.05 and 0.1 , with the exception of the two values for PLL(20)-g[11.2]-PEG(5) and PLL(20)-g[8.7]-dex(5.2), which show significantly higher $\mu$ values (close to that of the PLL backbone alone $(1 / g=0))$ and are presumably not in a brush conformation.

PLL(20)- $g$-dex(5.2) and PLL(20)-g-dex(10) copolymers behave similarly in the regime where $1 / g \geq$ ca. 0.15 , with their coefficients of friction slightly increasing in a fairly linear fashion. In the same regime $(1 / \mathrm{g} \geq \mathrm{ca}$. 0.15), PLL(20)-g-PEG(5) copolymers, instead, show a small decrease in the $\mu$ values. A general increase in $\mu$ values for PLL- $g$-dex copolymers with high $1 / g$ values (high number of grafted side chains) can be readily understood in terms of smaller molar concentrations. The fact that the variation of molar concentration (from ca. 1.5 to $4 \times 10^{-6} \mathrm{mmol} / \mathrm{mL}$ ) influences the lubricating properties of the PLL- $g$-dex copolymers exclusively in high-speed regime is closely related to the time intervals between the cycles of tribostress. Under the experimental conditions (pin-on-disk tribometry), the tribostress is applied in a cyclic fashion, the sliding speed (or rpm, to be more precise) setting the time scale during which excess polymers can replenish the area where initially adsorbed polymers may have been detached by tribostress. 
While with increasing $1 / g$, PLL-g-dex copolymers become heavier (higher molecular weight), and therefore, slower in returning to the surface after being scraped away from it during sliding contact, the molar concentration also decreases as the degree of grafted side chains increases, meaning that the excess polymer in bulk solution available to re-adsorb, i.e., "self-heal" the tribostressed area, decreases at the same time.

The slight decrease of $\mu$ values for PLL(20)- $g$-PEG(5) with increasing $1 / \mathrm{g}$ suggests that the adsorption kinetics of PLL(20)- $g$-PEG(5) copolymers, in contrast to that of PLL-g-dex copolymers, are sufficiently fast such that the variation of the molar concentration within the range of $1.5-4 \times 10^{-6} \mathrm{mmol} / \mathrm{mL}$ does not lead to any substantial disadvantages in the lubricating performance. Furthermore, the higher flexibility of PEG chains compared to that of dextran's stiffer sugar units might facilitate the rearrangement of the polymer molecules on the surface after desorption.

\subsubsection{Lubrication at Mixed Sliding/Rolling Contacts: MTM}

The lubricating properties of PLL- $g$-dex copolymer solutions under mixed sliding/rolling contact conditions have been characterized by means of MTM. The MTM experiments serve primarily to assess the lubricating properties of the copolymers under milder contact conditions than the pure sliding contacts characteristic of pin-on-disk tribometry. In addition, owing to the much higher speed range available from MTM (up to 2,500 $\mathrm{mm} \mathrm{s}^{-1}$ ), the formation of a full fluid-film lubricant layer by the polymer solutions can be investigated. Based on the pin-on-disk tribometry results above, two PLL- $g$-dex(5) copolymers, PLL(20)-g[3.4]-dex(5.2) and PLL(20)-g[5.3]-dex(5.2), which showed slightly different lubricating behavior in sliding contact, have been employed, together with other lubricant solutions. The results are shown in Fig. 13.

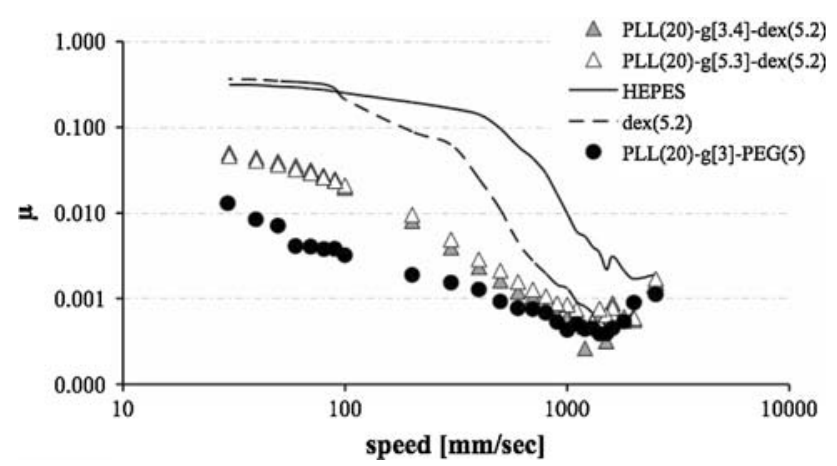

Fig. 13 Mixed sliding/rolling MTM results of two selected PLL(20)-g-dex(5.2) copolymers (steel balls versus glass disks, load: $10 \mathrm{~N}$, room temperature): coefficients of friction as a function of the sliding speed
The $\mu$ vs. speed plots shown in Fig. 13 revealed significant reduction of $\mu$ values in the high-speed regime (starting from ca. $200 \mathrm{~mm} \mathrm{~s}^{-1}$ ) for all aqueous lubricants employed, including polymer solutions and polymer-free HEPES buffer solution. The lowest $\mu$ value for HEPES buffer solution, $1.9 \times 10^{-3}$, which was achieved at the highest speed $\left(2,500 \mathrm{~mm} \mathrm{~s}^{-1}\right)$, for instance, represents approximately two orders of magnitude reduction compared with the highest $\mu$ value, 0.3 , observed at the lowest speed $\left(10 \mathrm{~mm} \mathrm{~s}^{-1}\right)$. This observation suggests that the entrainment of the base lubricant, water, is starting to contribute to the lubrication of this mixed sliding/rolling contacts $(\mathrm{SRR}=10 \%)$ in the high-speed regime. Nevertheless, all the polymer solutions investigated have been observed to further improve the lubricating properties, either in the high-speed regime alone or in both low- and high-speed regimes, depending on the type of polymers. For instance, the dextran solution revealed virtually identical $\mu$ vs. speed behavior to that of HEPES buffer in the low-speed regime ( $\left.\leq 100 \mathrm{~mm} \mathrm{~s}^{-1}\right)$, whereas a significant reduction in $\mu$ was observed in the high-speed regime, as mentioned above. It should be noted that at the $\operatorname{dex}(5.2)$ concentrations employed, no detectable increase in viscosity over that of pure HEPES solution could be detected in shear rheometry measurements (data not shown). Although dex(5.2) chains do not display noticeable surface-adsorption properties (Fig. 3), and thus are poor boundary-lubricating additives, they appear to facilitate the entrainment of aqueous lubricant due to their hydrophilic characteristics [52].

The two PLL-g-dex copolymer solutions showed a distinct reduction in $\mu$ values, both in low- and high-speed regimes, reflecting their adsorption onto the tribopair surfaces; compared with the dextran solution, the $\mu$ values are observed to be consistently lower over nearly the entire speed range investigated. This observation implies that the reduction in $\mu$ values in the high-speed regime is due not entirely to the formation of fluid-film, but also due partially to the improvement in the boundary lubrication properties. In fact, previous combined studies of the film thickness and frictional properties by means of ultra-thin film interferometry and MTM, respectively, have shown that the mixed lubrication mechanism is dominant for tribological contacts lubricated by PLL- $g$-PEG aqueous solutions [8]. In contrast to the results for sliding contacts, however, the two PLL-g-dex copolymers showed no noticeable difference in their lubricating properties, presumably because of the characteristics of the tribological contacts provided by MTM; first, the contact is much milder due to the dominance of rolling contact $(\mathrm{SRR}=10 \%)$ under these conditions, and thus the readsorption properties of the molecules are less critical. Second, the entrainment of the base lubricant, water, becomes more likely with increasing speed, even in the absence of additives. 
Finally, the lubricating performance of the PLL-g-dex copolymers under mixed sliding/rolling contact conditions was observed to be inferior to that of PLL(20)- $g$ [3.0]-PEG(5). This difference is more noticeable under low-speed conditions, and starts to diminish with increasing speed. PLL(20)- $g$ [6.5]-dex(10) and its PLL- $g$-PEG counterpart were also investigated by MTM, but they showed no difference in terms of the $\mu$-value trend, when compared to the PLL-g-dex(5) copolymers employed. These results have therefore been omitted in Fig. 13 for clarity purposes, to highlight the results discussed above.

\section{Conclusions}

Comb-like graft copolymers with dextran side chains (PLL-g-dex) have been developed and investigated as biomimetic lubricant additives in an aqueous environment. The synthesized copolymers have been shown to adsorb spontaneously onto negatively charged surfaces, similarly to PLL- $g$-PEG, and to behave as boundary lubricants, both in the pure sliding and in mixed sliding/rolling contact regimes.

Two series of PLL- $g$-dex copolymers, with varying grafting ratios and molecular weights of the dextran side chains (dex(5.2) and $\operatorname{dex}(10))$, were employed in this study and their lubricating capabilities compared to the PLL- $g$-PEG counterpart copolymers. The selection of the two dextrans was motivated by the desire to perform a fair comparison with the PLL- $g$-PEG copolymers: $\operatorname{dex}(5.2)$ is nearly identical with PEG(5) in terms of molecular weight (diffusion properties) and $\operatorname{dex}(10)$ and PEG(5) are comparable for the fully extended chain length (generable film thickness). The lubricating properties of the involved copolymers in the low-speed regime $\left(\leq \mathrm{ca} .10 \mathrm{~mm} \mathrm{~s}^{-1}\right)$ appeared to be related to the adsorption properties in equilibrium and not to be significantly influenced by the variation of molar concentration. Lubricating performances of PLL(20)- $g$-PEG(5) compared with PLL- $g$-dex copolymers are, in this regime, better over the entire range of grafting ratio: this might be attributed to the higher degree of hydration and flexibility of PEG chains compared to that of the sugar units in dextran.

In the high-speed regime ( $\geq \mathrm{ca} .10 \mathrm{~mm} \mathrm{~s}^{-1}$ ), all the PLL-g-dex copolymers, except for the case of $g$ [3.5], showed better lubricating performance than the PLL- $g$-PEG counterpart copolymers, but with a slightly different trend in the $\mu$ values with increasing $1 / g$; the selfhealing behavior of PLL-g-dex copolymers seems to be diminished, in fact, by decreasing molar concentration and increasing molecular weight. Nevertheless, such changes do not influence the performance of the PLL- $g$-PEG copolymers, presumably due to the greater flexibility of the PEG chains.
Acknowledgments We would like to thank Dr. Rowena Crockett of EMPA, Dübendorf, who made the Mini Traction Machine available to us. Effort sponsored by the Air Force Office of Scientific Research, Air Force Material Command, USAF, under grant number FA865505-1-3042. The U.S. Government is authorized to reproduce and distribute reprints for Government purpose notwithstanding any copyright notation thereon. The views and conclusions contained herein are those of the authors and should not be interpreted as necessarily representing the official policies or endorsements, either expressed or implied, of the Air Force Office of Scientific Research or the U.S. Government.

\section{References}

1. Havet, L., Blouet, J., Robbe Valloire, F., Brasseur, E., Slomka, D.: Tribological characteristics of some environmentally friendly lubricants. Wear 248, 140-146 (2001). doi:10.1016/S0043-1648 (00)00550-0

2. Willing, A.: Lubricants based on renewable resources-an environmentally compatible alternative to mineral oil products. Chemosphere 43, 89-98 (2001). doi:10.1016/S0045-6535(00) 00328-3

3. Uyama, Y., Tadokoro, H., Ikada, Y.: Low-frictional catheter materials by photo-induced graft polymerization. Biomaterials 12, 71-75 (1991). doi:10.1016/0142-9612(91)90135-W

4. Heuberger, M., Drobek, T., Spencer, N.D.: Interaction forces and morphology of a protein-resistant Poly(ethylene glycol) layer. Biophys. J. 88, 495-504 (2005). doi:10.1529/biophysj.104. 045443

5. Kenausis, G.L., Vörös, J., Elbert, D.L., Huang, N., Hofer, R., Ruiz-Taylor, L., Textor, M., Hubbell, J., Spencer, N.D.: Poly(Llysine)-g-Poly(ethylene glycol) layers on metal oxide surfaces: attachment mechanism and effects of polymer architecture on resistance to protein adsorption. J. Phys. Chem. B 104, 32983309 (2000). doi:10.1021/jp993359m

6. Lee, S., Müller, M., Ratoi-Salagean, M., Vörös, J., Pasche, S., De Paul, S.M., Spikes, H.A., Textor, M., Spencer, N.: Boundary lubrication of oxide surfaces by Poly(L-lysine)-g-poly(ethylene glycol) (PLL-g-PEG) in aqueous media. Tribol. Lett. 15, 231-239 (2003). doi:10.1023/A:1024861119372

7. Müller, M.: Aqueous Lubrication by Means of Surface-Bound Brush-Like Copolymers. ETH Zurich, Zurich (2005)

8. Müller, M., Lee, S., Spikes, H.A., Spencer, N.D.: The influence of molecular architecture on the macroscopic lubrication properties of the brush-like co-polyelectrolyte poly: (L-lysine)-g-poly(ethylene glycol) (PLL-g-PEG) adsorbed on oxide surfaces. Tribol. Lett. 15， 395-405 (2003). doi:10.1023/B:TRIL.0000003063. 98583.bb

9. Pasche, S., Paul, S.M., Vörös, J., Spencer, N.D., Textor, M.: Poly(L-lysine)-graft-poly(ethylene glycol) assembled monolayers on niobium oxide surfaces: a quantitative study of the influence of polymer interfacial architecture on resistance to protein adsorption by ToF-SIMS and in situ OWLS. Langmuir 19, 92169225 (2003). doi:10.1021/la034111y

10. Pasche, S., Textor, M., Meagher, L., Spencer, N.D., Griesser, H.J.: Relationship between interfacial forces measured by colloid-probe atomic force microscopy and protein resistance of poly/ethylen glycol)-grafted poly(L-lysine) adlayers on niobia surfaces. Langmuir 21, 6508-6520 (2005). doi:10.1021/la050386x

11. Blaettler, T.M., Pasche, S., Textor, M., Griesser, H.J.: High salt stability and protein resistance of poly(L-lysine)-g-poly(ethylene glycol) copolymers covalently immobilized via aldehyde plasma polymer interlayers on inorganic and polymeric substrates. Langmuir 22, 5760-5769 (2006). doi:10.1021/la034111y 
12. Han, S., Kim, C., Kwon, D.: Thermal degradation of poly(ethylenelycol). Polym. Degrad. Stab. 47, 203-208 (1995). doi:10.1016/ 0141-3910(94)00109-L

13. Han, S., Kim, C., Kwon, D.: Thermal/oxidative degradation and stabilization of polyethylene glycol. Polymer (Guildf.) 38, 317-323 (1997). doi:10.1016/S0032-3861(97)88175-X

14. Holland, N.B., Qiu, Y., Ruegsegger, M., Marchant, R.E.: Biomimetic engineering of non-adhesive glycocalyx-like surfaces using oligosaccharide surfactant polymers. Nature 392, 799-801 (1998). doi:10.1038/33894

15. McArthur, S.L., McLean, K.M., Kingshott, P., John, H.A.W., Chatelier, R.C., Griesser, H.J.: Effect of polysaccharide structure on protein adsorption. Colloids Surf. B Biointerfaces 17, 37-48 (2000). doi:10.1016/S0927-7765(99)00086-7

16. Piehler, J., Brecht, A., Hehl, K., Gauglitz, G.: Protein interactions in covalently attached dextran layers. Colloids Surf. B Biointerfaces 13, 325-336 (1999). doi:10.1016/S0927-7765(99)00046-6

17. Qiu, Y., Zhang, T., Ruegsegger, M., Marchant, R.E.: Novel nonionic oligosaccharide surfactant polymers derived from poly(vinylamine) with pendant dextran and hexanoyl groups. Macromolecules 31, 165-171 (1998). doi:10.1021/ma9707401

18. Sen Gupta, A., Wang, S., Link, E., Anderson, E.H., Hofmann, C., Lewandowski, J., Kottke-Marchant, K., Marchant, R.E.: Glycocalyx-mimetic dextran-modified poly(vinyl amine) surfactant coating reduces platelet adhesion on medical-grade polycarbonate surface. Biomaterials 27, 3084-3095 (2006). doi:10.1016/j.bio materials.2006.01.002

19. Naka, M., Morita, Y., Ikeuchi, K.: Influence of proteoglycan contents and of tissue hydration on the frictional characteristics of articular cartilage. Proc. Inst. Mech. Eng. [H] 219, 175-182 (2005). doi:10.1243/095441105X34220

20. Pickard, J., Ingham, E., Egan, J., Fisher, J.: Investigation into the effect of proteoglycan molecules on the tribological properties of cartilage joint tissues. Proc. Inst. Mech. Eng. 212, 177-182 (1998)

21. Bansil, R., Turner, B.S.: Mucin structure, aggregation, physiological functions and biomedical applications. Curr. Opin. Colloid Interface Sci. 11, 164-170 (2006). doi:10.1016/j.cocis. 2005.11.001

22. Dalsin, J.L.: Mussel adhesive-inspired surface modification for the preparation of nonfouling biomaterials. Ph.D. Dissertation, Northwestern University (2004)

23. Hahn Berg, C.I., Lindh, L., Arnebrant, T.: Intraoral lubrication of PRP-1, Statherin and Mucin as Studied by AFM. Biofouling 20, 65-70 (2004). doi:10.1080/08927010310001639082

24. Lee, S., Müller, M., Rezwan, K., Spencer, N.D.: Porcine gastric mucin (PGM) at the water/poly(dimethylsiloxane) (PDMS) interface: influence of $\mathrm{pH}$ and ionic strength on its conformation, adsorption, and aqueous lubrication properties. Langmuir 21, 8344-8353 (2005). doi:10.1021/la050779w

25. Choi, S.W., Sato, Y., Akaike, T., Maruyama, A.: Preparation of cationic comb-type copolymer having guanidino moieties and its interaction with DNAs. J. Biomater. Sci. Polym. Ed. 15, 10991110 (2004). doi:10.1163/1568562041753089

26. Ferdous, A., Akaike, T., Maruyama, A.: Inhibition of sequencespecific protein-DNA interaction and restriction endonuclease cleavage via triplex stabilization by poly(L-lysine)-graft-dextran copolymer. Biomacromolecules 1, 186-193 (2000). doi:10.1021/ bm9900141

27. Ferdous, A., Watanabe, H., Akaike, T., Maruyama, A.: Combtype copolymer: stabilization of triplex DNA and possible application in antigene strategy. J. Pharm. Sci. 87, 1400-1405 (1998). doi:10.1021/js980066g

28. Ferdous, A., Watanabe, H., Akaike, T., Maruyama, A.: Poly(Llysine)-graft-dextran copolymer: amazing effects on triplex stabilization under physiological $\mathrm{pH}$ and ionic conditions (in vitro).
Nucleic Acids Res. 26, 3949-3954 (1998). doi:10.1093/ nar/26.17.3949

29. Maruyama, A., Watanabe, H., Ferdous, A., Katoh, M., Ishihara, T., Akaike, T.: Characterization of interpolyelectrolyte complexes between double-stranded DNA and polylysine comb-ype copolymers having hydrophilic side chains. Bioconjugate Chem. 9, 292-299 (1998)

30. Sato, Y., Kobayashi, Y., Kamiya, T., Watanabe, H., Akaike, T., Yoshikawa, K., Maruyama, A.: The effect of backbone structure on polycation comb-type copolymer/DNA interactions and the molecular assembly of DNA. Biomaterials 26, 703-711 (2005). doi:10.1016/j.biomaterials.2004.03.018

31. Torigoe, H., Ferdous, A., Watanabe, H., Akaike, T., Maruyama, A.: Poly(L-lysine)-graft-dextran copolymer promotes pyrimidine motif triplex DNA formation at physiological pH. J. Biol. Chem. 274, 6161-6167 (1999). doi:10.1074/jbc.274.10.6161

32. Frazier, R.A., Matthijs, G., Davies, M.C., Roberts, C.J., Schacht, E., Tendler, S.J.B.: Characterization of protein-resistant dextran monolayers. Biomaterials 21, 957-966 (2000). doi:10.1016/ S0142-9612(99)00270-7

33. Griesser, H.J., Hartley, P.G., McHarthur, S.L., McLean, K.M., Meagher, L., Thissen, H.: Interfacial properties and protein resistance of nano-scale polysaccharide coatings. Smart Mater. Struct. 11, 652-661 (2002). doi:10.1088/0964-1726/11/5/305

34. Martwiset, S.: Nonfouling characteristics of dextran-containing surfaces. Langmuir 22, 8192-8196 (2006). doi:10.1021/la06 $1064 \mathrm{~b}$

35. Mc Lean, K.M., Johnson, G., Chatelier, R.C., Beumer, G.J., Steele, G., Griesser, H.J.: Method of immobilization of carboxymethyl-dextran affects resistance to tissue and cell colonization. Colloids Surf. B Biointerfaces 18, 221-234 (2000). doi:10.1016/S0927-7765(99)00149-6

36. Österberg, E., Bergström, K., Holmberg, K., Schuman, T.P., Riggs, J.A., Burns, N.L., Van Alstine, J.M., Harris, J.M.: Proteinrejecting ability of surface-bound dextran in end-on and side-on configurations: comparison to PEG. J. Biomed. Mater. Res. 29, 741-747 (1995). doi:10.1002/jbm.820290610

37. Snabre, P., Grossmann, G.H., Mills, P.: Effects of dextran polydispersity on red blood cell aggregation. Colloid Polym. Sci. 263, 478-483 (1985). doi:10.1007/BF01458338

38. Wang, M.S., Palmer, L.B., Schwartz, J.D., Razatos, A.: Evaluating protein attraction and adhesion to biomaterials with the atomic force microscope. Langmuir 20, 7753-7759 (2004). doi: 10.1021/la049849+

39. Liu, W., Yu, D., Yang, M.: Blood compatibility of thermoplastic polyurethane membrane immobilized with water-soluble chitosan/dextran sulfate. Colloids Surf. B Biointerfaces 44, 82-92 (2005). doi:10.1016/j.colsurfb.2005.05.015

40. Perrino, C., Lee, S., Spencer, N.D.: A biomimetic alternative to PEG as an antifouling coating: resistance to non-specific protein adsorption of poly(L-lysine)-graft-dextran. Langmuir 24, 88508856 (2008). doi:10.1021/la800947z

41. Green, R.J., Davies, M.C., Roberts, C.J., Roberts, S.J.B.J.: J. Biomed. Mater. Res. 42, 165-171 (1998). doi :10.1002/(SICI) 1097-4636(199811)42:2<165::AID-JBM1>3.0.CO;2-N

42. Lee, S., Iten, R., Müller, M., Spencer, N.D.: Influence of molecular architecture on the adsorption of poly(ethylene oxide)poly(propylene oxide)-poly(ethylene oxide) (PEO-PPO-PEO) on PDMS surfaces and implications for aqueous lubrication. Macromolecules 37, 8349 (2004). doi:10.1021/ma049076w

43. Vörös, J., Ramsden, J.J., Csucs, G., Szendro, I., De Paul, S.M., Textor, M., Spencer, N.D.: Optical grating coupler biosensors. Biomaterials 23, 3699-3710 (2002). doi:10.1016/S0142-9612(02) 00103-5

44. Hook, F., Vörös, J., Rodahl, M., Kurrat, R., Böni, P., Ramsden, J.J., Textor, M., Spencer, N.D., Tengvall, P., Gold, J., 
Kasemo, B.: A comparative study of protein adsorption on titanium oxide surfaces using in situ ellipsometry, optical waveguide lightmode spectroscopy, and quartz crystal microbalance/dissipation. Colloids Surf. B Biointerfaces 24, 155-170 (2002). doi: 10.1016/S0927-7765(01)00236-3

45. Kurrat, R., Textor, M., Ramsden, J.J., Böni, P., Spencer, N.D.: Instrumental improvements in optical waveguide light mode spectroscopy for the study of biomolecule adsorption. Rev. Sci. Instrum. 68, 2172-2176 (1997). doi:10.1063/1.1148069

46. Rixman, M.A., Dean, D., Ortiz, C.: Nanoscale intermolecular interactions between human serum albumin and low grafting density surfaces of poly(ethylene oxide). Langmuir 19, 9357-9372 (2003). doi:10.1021/la0340571

47. Kawaguchi, T., Hasegawa, M.: Structure of dextran-magnetite complex: relation between conformation of dextran chains covering core and its molecular weight. J. Mater. Sci. Mater. Med. 11, 31-35 (2000). doi:10.1023/A:1008933601813

48. Görisch, S.M., Wachsmuth, M., Fejes Toth, K., Lichter, P., Rippe, K.: Histone acetylation increases chromatin accessibility. J. Cell Sci. 118, 5825-5834 (2005). doi:10.1242/jcs.02689
49. Kawaguchi, S., Imai, G., Suzuki, J., Miyahara, A., Kitano, T., Ito, K.: Aqueous solution properties of oligo- and poly(ethylene oxide) by static light scattering and intrinsic viscosity. Polymer (Guildf.) 38, 2885-2891 (1997). doi:10.1016/S0032-3861(96) 00859-2

50. Lee, S., Müller, M., Heeb, R., Zürcher, S., Tosatti, S., Heinrich, M., Amstad, F., Pechmann, S., Spencer, N.D.: Self-healing behavior of a polyelectrolyte-based lubricant additive for aqueous lubrication of oxide materials. Tribol. Lett. 24, 217-223 (2006). doi:10.1007/s11249-006-9121-9

51. Tyrode, E., Johnson, C., Kumpulainen, A., Rutland, M.W., Claesson, P.M.: Hydration state of nonionic surfactant monolayers at the liquid/vapor interface: structure determination by vibrational sum frequency spectroscopy. J. Am. Chem. Soc. 127, 16848-16859 (2005). doi:10.1021/ja053289z

52. Smeeth, M., Spikes, H.A., Gunsel, S.: The formation of viscous surface films by polymer solutions: boundary or elastohydrodynamic lubrication? Tribol. Trans. 39, 720-725 (1996). doi: 10.1080/10402009608983589 